\title{
SNUH SEOUL NATIONAL UNIVERSITY
}

Correlation and agreement between observed ETCO2 and predicted ETCO2 calculated from EEG signals in out-of-hospital cardiac arrest: a pilot tiral

Ki Hong Kim MD, Sang Do Shin MD, Tae Han Kim MD, Ki Jeong Hong MD, Hee Chan Kim PhD, Hee Jin Kim MS

Department of Emergency Medicine, Seoul National University Hospital

Department of Bioengineering, Seoul National University Hospital

\section{Backgjound}

- Brain cell viability was not measured directly during cardiopulmonary resuscitation (CPR) in out-of-hospital cardiac arrest (OHCA)

- We developed the EEG-based Brain Resuscitation Index (EBRI) which is an expected value of end-tidal CO2 calculated from EEG

- We compared the observed ETCO2 and EBRI during CPR.

\section{Methods}

- OHCA patients who received CPR at one tertiary emergency department were enrolled for this study - Convenient sampling \& exclude if head trauma or deformity

- All CPR procedures were provided without any change, but the single EEG electrode was attached on the forehead of OHCA patients

- The EEG signals were transmitted via designed wireless Bluetooth technology to and independent mobile computer
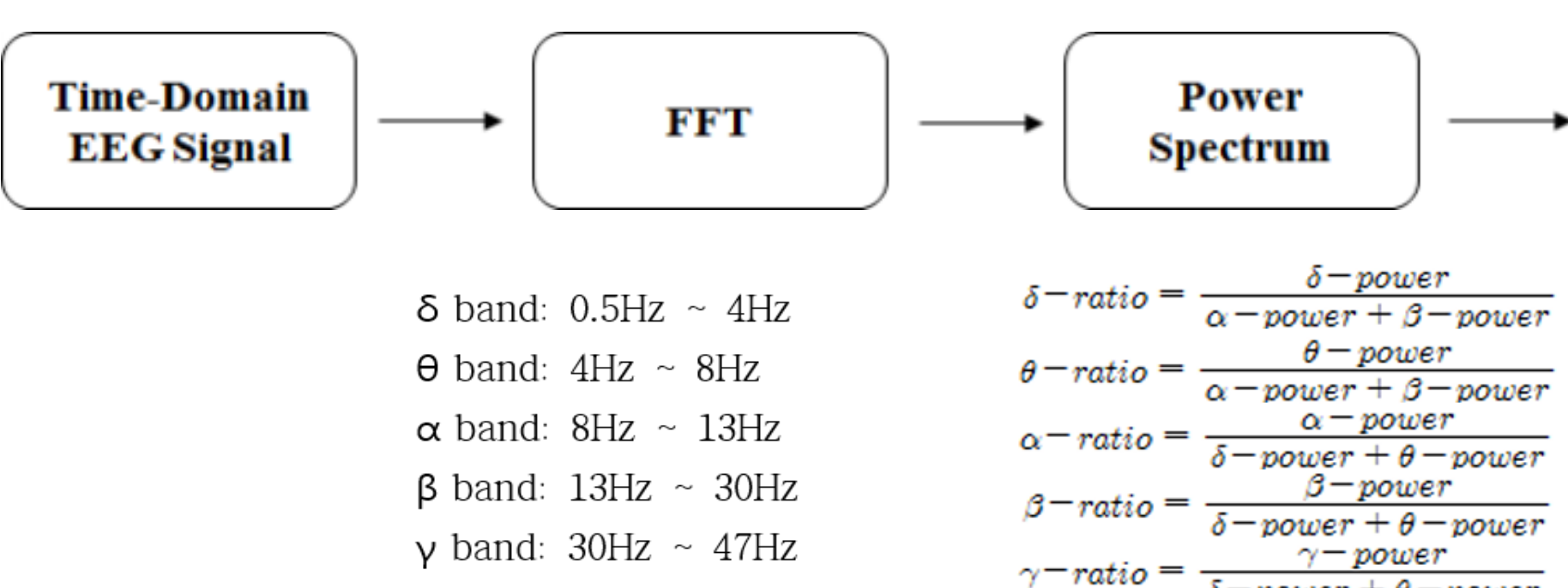

- Computation formula to calculate the expected ETCO2 using multiple EEG signal values ( 2 model )

EBRI (Expected ETCO2) $=\mathbf{a}+\mathbf{b} 1 \times$ BSR $+\mathbf{b 2} \times$ BetaR + b3 $\times$ SynchFastSlow + b4 $\times$ DeltaR

* BSR : Beta-SEF95 ratio, BetaR : $\beta$-ratio, DeltaR $: \delta$-ratio

It was developed in previous animal study and validated for human EEG in former observation study

- Data collection was conducted till return of spontaneous circulation or termination of CPR

- Each data was continuously collected by 0.5 second intervals and extremely deviated values such as observed ETCO2 level lower than 5 or higher than 60 , were excluded

- After 1-minute averaging, Pearson Correlation Analysis and Bland-Altman agreement plot between observed ETCO2 and EBRI were performed

-We tested the repeated ANOVA for change of trend

\section{Resulits}

- Total 5 patients with EEG signals during CPR of eligible 13 OHCA patients were enrolled and analyzed (November 2017 - April 2018)

- Total 4989 observed ETOC2 and EBRI values were collected after data cleaning

- Average ETCO2 value per minute were used for correlation and agreement analysis

- Positive correlation ( Pearson correlation coefficient $0.53, p$-value $<0.01$ ) was found in EBRI model 2

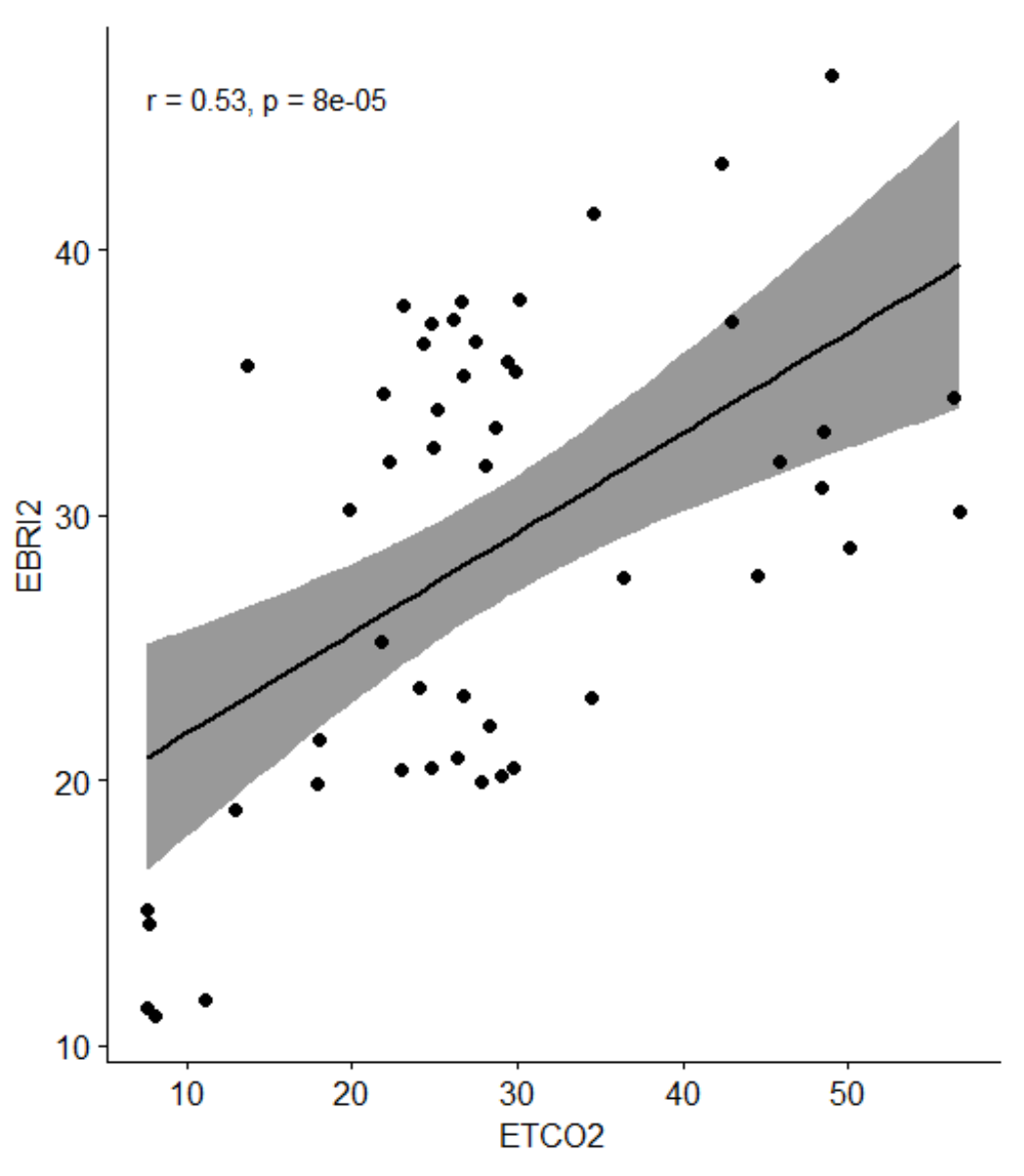

\section{Conclusion}

- We found positive correlation and higher agreement between observed ETCO2 and expected ETCO2 from EEG signals during CPR in OHCA patients

- The agreement by the Bland-Altman plot showed only $4 / 48(8.3 \%)$ observations were not within agreement (in both model)

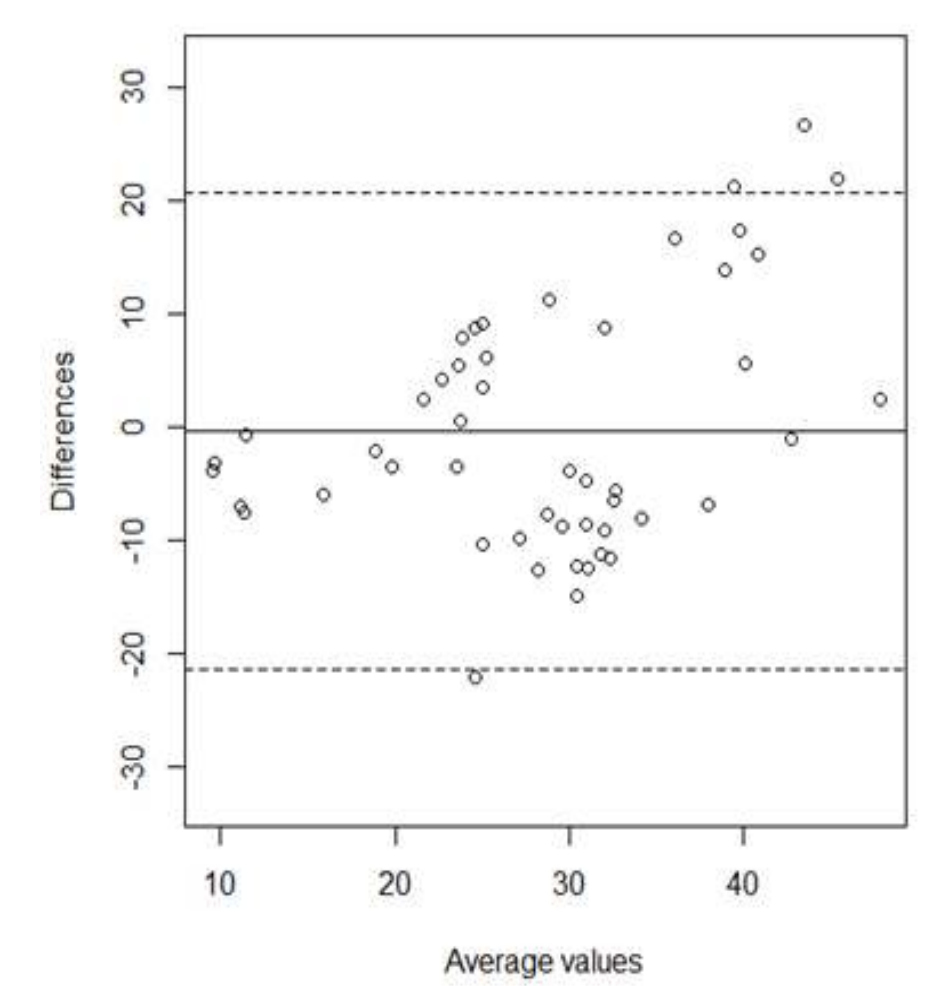

- There was no significant difference in trend according to CPR duration between observed ETCO2 and EBRI

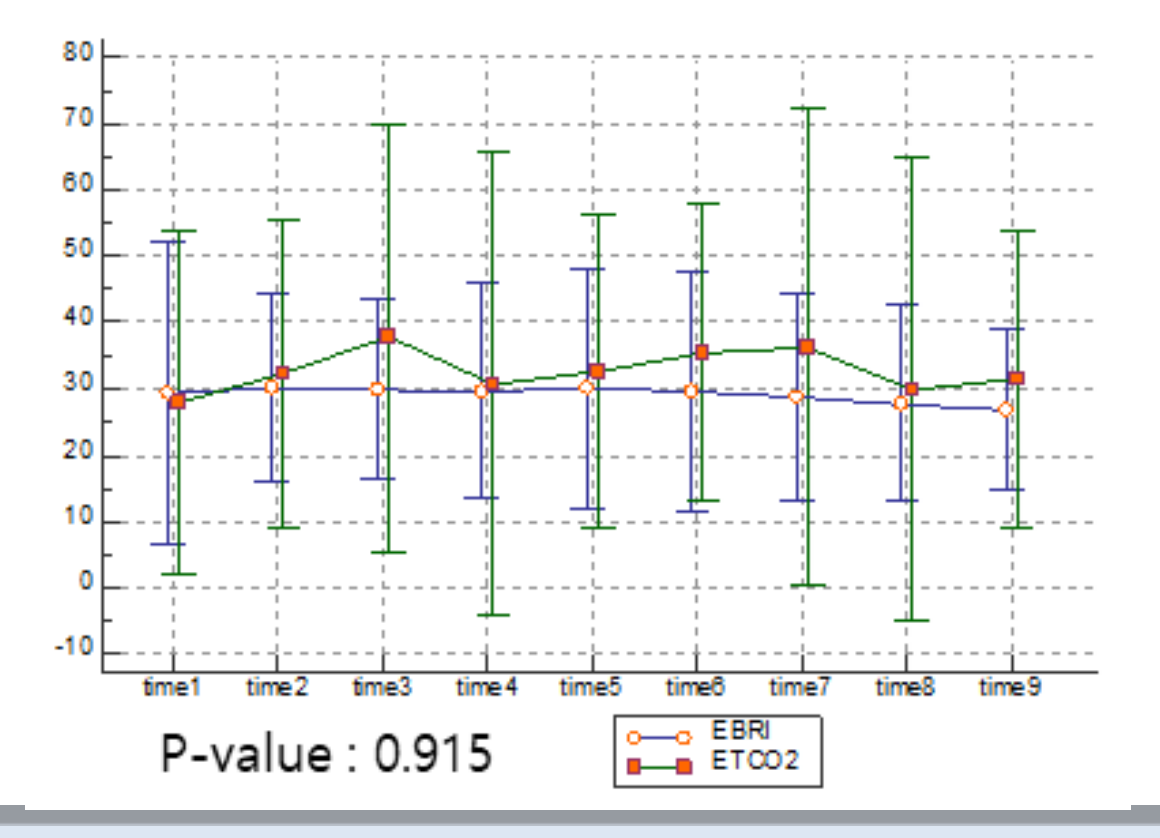

P-value:0.915 0

(1) 